\title{
Biochemical markers in differentiating gallstones pancreatitis from non-gallstone pancreatitis
}

\author{
Masoud Alghamdi ${ }^{1 *}$, Afnan Hassan Alrashdi², Addi Hatem Almaghamsi ${ }^{3}$, Atif Essa Alamri ${ }^{3}$, \\ Bushra Ahmad Abdulrashid ${ }^{3}$, Zaini Mohammedsaeed Sabban ${ }^{3}$, Abdullah Lafi Alhassan, \\ Fahad Salem Alanazi ${ }^{5}$, Sulaiman Abdelaziz Abanmi ${ }^{6}$, Fayez Dhyefallah Alghamdi ${ }^{7}$, \\ Layla Khalid Alshammari ${ }^{8}$, Al-Hassan Mohammed Ba Hussein?
}

\author{
${ }^{1}$ Department of General Surgery, King Fahad Hospital, Jeddah, Saudi Arabia \\ ${ }^{2}$ College of Medicine Al-Qunfudhah, Umm Al-Qura University, Al-Qunfudhah, Saudi Arabia \\ ${ }^{3}$ Department of Emergency Medicine, Almadinah General Hospital, Medina, Saudi Arabia \\ ${ }^{4}$ Department of General Surgery, Prince Mutaib bin Abdulaziz Hospital, Skaka, Saudi Arabia \\ ${ }^{5}$ College of Medicine, Shaqra University, Shaqra, Saudi Arabia \\ ${ }^{6}$ College of Medicine, King Saud bin Abdulaziz University for Health Sciences, Riyadh, Saudi Arabia \\ ${ }^{7}$ College of Medicine, Baha University, Baha, Saudi Arabia \\ ${ }^{8}$ College of Medicine, Princess Nourah Bint Abdul Rahman University, Riyadh, Saudi Arabia \\ ${ }^{9}$ College of Medicine, MAHSA University, Kuala Lumpur, Malaysia
}

Received: 15 December 2020

Accepted: 31 December 2020

*Correspondence:

Dr. Masoud Alghamdi,

E-mail: drmasoud2004@yahoo.com

Copyright: @ the author(s), publisher and licensee Medip Academy. This is an open-access article distributed under the terms of the Creative Commons Attribution Non-Commercial License, which permits unrestricted non-commercial use, distribution, and reproduction in any medium, provided the original work is properly cited.

\section{ABSTRACT}

Acute pancreatitis has many causes; however, the most common cause of pancreatitis is gallstones globally. We have searched for relevant studies in this field aiming at establishing a short review about the usefulness of biomarkers in the diagnosis of acute pancreatitis and the relevant biomarkers and tests that can differentiate acute gallstone pancreatitis from other etiologies. It is worth mentioning that for the diagnosis and prognosis of biliary pancreatitis, radiological and laboratory diagnosis plays a major role in this field. Three biomarkers including serum amylase, lipase, and trypsinogen are the most important and most frequently noticed biomarkers in association with acute pancreatitis. Serum amylase has a specificity rate of $95 \%$ and a sensitivity of $61 \%$ when it measures more than 1000 IU/l which is three times as high as the normal level. However, it can be also found in other intrabdominal inflammatory conditions, and therefore, it cannot be used alone in the diagnosis. It has been reported that the specificity and sensitivity were $95 \%$ and $94 \%$, respectively, for detecting high trypsinogen-2 levels in the patients' urinary samples, which were indicative of acute pancreatitis. For the diagnosis of biliary pancreatitis, liver function tests should be assessed. Although they might be specific, they are not always diagnostic in some cases, and therefore, other approaches for detecting gallstones as ultrasonography and MRCP should be considered together with the liver enzymes for an appropriate diagnosis.

Keywords: Pancreatitis, Gallstone, Biomarker; Trypsinogen, Liver functions

\section{INTRODUCTION}

Acute pancreatitis has many causes; however, the most common cause of pancreatitis is gallstones globally. This etiology has been found to occur in $50 \%$ of the patients that suffer from acute pancreatitis in the western nations while acute pancreatitis itself accounts for 4.8-24.2 per 100,000 cases in these countries. ${ }^{1-3}$ In the United States, 
acute pancreatitis affects around 80,000 patients, which are equal to 17 per $100,000 .^{4}$ A yearly incidence that ranges between $5-80$ per 100,000 has also been estimated in the Japanese population. ${ }^{5,6}$ In patients with acute biliary pancreatitis, spontaneous recovery usually occurs in $15-30 \%$ of the patients, however, in some cases, patients develop severe sequelae of pancreatitis that requires special attention for appropriate management. ${ }^{7}$ Moreover, if left untreated, many complications following acute biliary pancreatitis as necrosis, hemorrhage, abscess, and pseudocyst formation, in addition to other disorders of systemic involvement as pleural effusion, renal impairment, adult respiratory distress syndrome, and other complications that usually require intensive care unit admission for management. ${ }^{7,8}$

Many risk factors as, gender and the size of the stone may be associated with the development of biliary pancreatitis, and therefore, the identification of such risk factors might be important for the early detection and management of acute pancreatitis. Interestingly, acute pancreatitis is more common in male patients while it has been noticed that the development of gallstones is more common in female patients. ${ }^{9}$ The pathophysiology of acute pancreatitis has not been fully comprehended, yet, however, many theories have been proposed. Moreover, many etiologies have been reportedly associated with the development of acute pancreatitis. Premature activation of trypsinogen to trypsin within the pancreas is thought to play a major role in the inflammation of the pancreatic tissue. ${ }^{10,11}$ Additionally, looking at its unique function of activating other pancreatic enzymes, activated trypsinogen also plays a major role in the process of autodigestion found in acute pancreatitis. $^{12}$ For the diagnosis of pancreatitis, many serum biomarkers might be helpful. These include serum amylase, serum lipase, and serum and urinary trypsinogen. These biomarkers are of great value in the management and diagnosis of acute pancreatitis and all of them have variable efficacies that should be considered when initiating the diagnosis.

Acute pancreatitis is considered a fatal disease although many interventional approaches have been proposed. The estimated mortality rate for acute pancreatitis has been estimated to be 2-7\%. ${ }^{13-16}$ Two major factors are considered as the determinant of the prognosis and severity of the diseases like organ failure and the presence of pancreatic necrosis. It has also been estimated that around $50 \%$ of the patients with acute pancreatitis usually die within the first one to two weeks, which may be induced by the presence of an underlying organ failure. In addition to the multiple complications that may occur following biliary pancreatitis, an estimated risk ratio of $32-61 \%$ was found for acute biliary pancreatitis recurrence. ${ }^{14,15}$ Therefore, it might be crucial in identifying the proper etiology of acute pancreatitis for proper management which may be dependent on the etiology. We have searched for relevant studies in this field aiming at establishing a short review about the usefulness of biomarkers in the diagnosis of acute pancreatitis and the relevant biomarkers and tests that can differentiate acute gallstone pancreatitis from other etiologies.

Extensive literature search of the Medline, Cochrane, and EMBASE databases was performed on 18 December 2020 using the medical subject headings (MeSH) or a combination of all possible related terms. Studies discussing the usefulness of biomarkers in the diagnosis of acute pancreatitis and the relevant biomarkers and tests that can differentiate acute gallstone pancreatitis from other etiologies; were screened for relevant information. We did not pose any limits on date, language, or publication type.

\section{Differentiating gallstones from non-gallstones acute pancreatitis}

Studies suggest that acute biliary pancreatitis diagnosis should be established if the patient has a history that mimics the pain of biliary colic. ${ }^{17,18}$ Although acute biliary pancreatitis is deemed the commonest type of pancreatitis, when initiating the diagnosis and management, other causes of acute pancreatitis should be considered. For instance, prolonged alcohol consumption is also another common cause of acute pancreatitis. Other etiologies include the consumption of certain medications that can cause pancreatic toxicity, infectious diseases, genetic disorders, postoperative cause, and other causes as surgical operations involving the pancreas, or the bile duct which can cause pancreatitis from an underlying injury. ${ }^{18-20}$ Although pancreatic biomarkers play an important role in initiating the appropriate diagnosis, carefully approaching the patient by obtaining a proper history and physical examination would be the key-role for a rightful diagnosis. For the diagnosis and prognosis of biliary pancreatitis, radiological and laboratory diagnosis also play a major role in this field. ${ }^{21,22}$

\section{DIAGNOSTIC BIOMARKERS OF ACUTE PANCREATITIS}

\section{Serum amylase}

Elevated levels of the serum amylase is an indicator of acute pancreatitis on the condition that the rise would be three times higher than the normal limits. It is considered as the fastest marker to rise in the patient's serum as it rises 12 hours within the onset of acute pancreatitis symptoms then it rapidly falls in the next four to five days. ${ }^{23}$ However, in $19-32 \%$ of patients with acute pancreatitis that are admitted to the hospital, serum amylase may show normal levels which may be attributable to delayed admission by the patient or the presence of an underlying exocrine insufficiency which may be attributable to chronic alcohol consumption. ${ }^{24,25}$ Additionally, the presence of other conditions as hypertriglyceridemia in the affected patient's serum may interfere with the appropriate interpretation of abnormal serum amylase levels masking these high levels, and 
therefore, this should be considered by administration of lipid-lowering drugs by the patient before measuring the amylase levels to obtain the right results. ${ }^{26}$ Although serum amylase levels are good indicators of acute pancreatitis, it can also be found elevated in other conditions as in other intra-abdominal inflammatory disorders, salivary gland diseases, and renal impairment because the kidneys cannot tolerate the excretion of the excess amounts of serum amylase leading to macroamylasaemia as serum amylase has been found conjugated with circulating compounds as polysaccharides and immunoglobulins leading to the formation of larger molecular weight compounds that are hard to excrete. ${ }^{26}$ Serum amylase sensitivity and specificity of detection of acute pancreatitis are hugely dependant on the specified threshold. A review by Yadav et al showed that a specificity rate of $95 \%$ and a sensitivity one of $61 \%$ for serum amylase can be found when it measures more than 1000 IU/l which is three times as high as the normal level. ${ }^{26}$ Therefore, other adjuvant approaches should be used when approaching the diagnosis of acute pancreatitis.

\section{Serum lipase}

The advantage of serum lipase over amylase is that it remains in the patient's serum for a longer period (8-14 days), therefore, it can be used for the diagnosis of acute pancreatitis in patients with delayed hospital admission. Because serum lipase activities are four times as high as these of serum amylase, it is less likely to be affected by the presence of chronic pancreatic insufficiency. ${ }^{27} \mathrm{~A}$ review by Werner et al suggests that serum lipase, whenever available, should be preferred to serum amylase according to recent United Kingdom guidelines for managing pancreatitis. ${ }^{28}$ Despite these advantages, elevated serum lipase levels are not always specific to acute pancreatitis. Similar to amylase, it can also be detected in other intraabdominal inflammatory conditions and renal impairment. Although hypertriglyceridemia does not interfere with the high levels of serum lipase, high levels can be found after the adminstration of certain drugs as frusemide. Besides, previous reports show that serum lipase has a better predicitve value for acute pancratitis. The estimated specificity for a $600 \mathrm{IU} / 1$ elvation in the serum lipase levels is equal to or more than $95 \%$ while the estimated sensitivity ranges between 55 $100 \% .^{26,29}$

\section{Serum and urinary trypsinogen}

Trypsinogen is a pro-enzyme that is cleaved in the presence of duodenal enterokinase or by the presence of inadequate amounts of trypsin, which results in the conversion of trypsinogen into trypsin, by positive feedback. This process results in the formation of trypsinogen activation peptide (TAP), and active $24 \mathrm{kDa}$ protease trypsin. There are two major isoenzymes of trypsinogen; 1 and 2 . Trypsinogen-2 exits in acute pancreatitis in high serum levels. ${ }^{30}$ Genetic predisposition plays a major role in trypsinogen dysfunction and can lead to the formation of pancreatitis. ${ }^{12}$ Moreover, high serum calcium levels may also predispose to the development of pancreatitis as calcium intervenes against the autolysis of trypsin. ${ }^{31}$ Within a few hours to three days from the initiation of acute pancreatitis, high levels of serum and urinary trypsinogen- 2 can be detected in the affected patient. In patients with acute pancreatitis following endoscopic retrograde cholangiopancreatography, high levels of this iso-enzyme could be detected in the serum within one hour. ${ }^{32}$ Kemppainen et al conducted a retrospective study of 500 patients presenting with symptoms of acute pancreatitis to determine the positive predictive value of trypsinogen-2. ${ }^{33}$ The authors reported that the estimated specificity and sensitivity were $95 \%$ and 94\%, respectively, for detecting high trypsinogen-2 levels in the patients' urinary samples which were indicative of acute pancreatitis. The sensitivity and specificity of urinary trypsinogen-2 were higher than the serum levels of amylase and lipase in the determination of acute pancreatitis. Moreover, a negative predictive value of $99 \%$ has been previously estimated for urinary trypsinogen- 2 in diagnosing acute pancreatitis. ${ }^{29}$ Therefore, it has been estimated that trypsinogen-2 levels might be more efficacious than serum levels of amylase and lipase in detecting acute pancreatitis.

\section{Diagnosis and identification of acute gallstone pancreatitis}

The first step for initiating the diagnosis of acute biliary pancreatitis is an abdominal ultrasound for the detection of stones blocking the biliary ducts and canaliculi starting from the gall bladder to the common bile duct. Ultrasound imaging has been reported to have a sensitivity of $95 \%$ of any underlying gallstones on the condition that the case was not complicated. However, this rate begins to decrease reaching up to $67-87 \%$ when the case is complicated with acute pancreatitis as a result of bowel and/or biliary distensions. ${ }^{34}$ Although the sensitivity of ultrasound might be high, previous studies also showed that the sensitivity for detection of gallstones in the common bile duct is hugely variable ranging between $25-90 \%$. In addition to the aforementioned biomarkers, other tests and markers help to differentiate between biliary and non-biliary pancreatitis. Liver function tests and enzymes, for instance, are hugely useful in the diagnosis of gallstones pancreatitis. A previous meta-analysis showed that a triple time increase in the levels of serum alanine transaminase, which is nearly equal to or more than $60 \mathrm{IU} / 1$ within 48 hours from the initiation of symptoms, is a good indicator of the presence of gall stones and biliary obstruction, in addition to the presence of acute pancreatitis with an estimated positive predictive value of $95 \% .{ }^{35}$ The same results were also confirmed by a previous study which supports the hypothesis. ${ }^{36}$ On the other hand, another study showed that normal liver enzymes were detected in $10-15 \%$ of the patients with acute gallstones pancreatitis. ${ }^{37}$ Therefore, liver enzymes should not be always used for the diagnosis 
of biliary pancreatitis. Other imaging procedures as magnetic resonance cholangiography (MRCP) has also shown favourable outcomes regarding the diagnosis of gallstones presence and biliary obstruction with an estimated specificity, sensitivity, positive predictive value, and a negative predictive value of 96-100\%, 84$95 \%, 91-100 \%$, and $92-98 \%$, respectively. ${ }^{38-41}$ However, previous studies said that this role has not been evidenced, yet, in the diagnosis of associated pancreatitis. This tool might be highly favorable for confirmation of the diagnosis of severely ill patients with acute pancreatitis that has been previously diagnosed with biomarkers and abdominal ultrasonography. Endoluminal ultrasonography has also been used as a useful technique for the detection of gallstones that is similar to the MRCP. ${ }^{42}$

\section{CONCLUSION}

In this review, we have discussed the relevant biomarkers that can be used in the diagnosis of actual pancreatitis. Three biomarkers including serum amylase, lipase, and trypsinogen are the most important and most frequently noticed biomarkers in association with acute pancreatitis. Although serum and urinary trypsinogen levels might be the best markers for establishing the diagnosis of acute pancreatitis, it is estimated that all of these biomarkers should be investigated together with other diagnostic approaches. For the diagnosis of biliary pancreatitis, liver function tests should be assessed. Although they might be specific, they are not always diagnostic in some cases, and therefore, other approaches for detecting gallstones as ultrasonography and MRCP should be also considered together with the liver enzymes for an appropriate diagnosis.

\section{Funding: No funding sources \\ Conflict of interest: None declared \\ Ethical approval: Not required}

\section{REFERENCES}

1. Srettabunjong S, Limgitisupasin W. Severe acute hemorrhagic pancreatitis secondary to cholelithiasis as a rare cause of sudden unexpected death in medico-legal case: A case report. Medicine. 2016; 95(34):e4680.

2. Vidarsdottir H, Möller PH, Vidarsdottir H, Thorarinsdottir H, Björnsson ES. Acute pancreatitis: a prospective study on incidence, etiology, and outcome. Eur J gastroenterol hepatol. 2013;25(9): 1068-75.

3. Moreau JA, Zinsmeister AR, Melton LJ, DiMagno EP. Gallstone pancreatitis and the effect of cholecystectomy: a population-based cohort study. Mayo Clin Proc. 1988;63(5):466-73.

4. Eland IA, Sturkenboom MJ, Wilson JH, Stricker BH. Incidence and mortality of acute pancreatitis between 1985 and 1995. Scand J gastroenterol. 2000;35(10): 1110-6.
5. Sekimoto M, Takada T, Kawarada Y. JPN Guidelines for the management of acute pancreatitis: epidemiology, etiology, natural history, and outcome predictors in acute pancreatitis. J Hepatobiliary Pancreat Surg. 2006;13(1):10-24.

6. Imamura M. Epidemiology of acute pancreatitis-incidence by etiology, relapse rate, cause of death and long-term prognosis. Nihon Rinsho Japanese $\mathbf{J}$ Clin Med. 2004;62(11):1993-7.

7. Visconti M, Rabitti PG, Uomo G, Giannattasio F, Varriale M, Russo C. The multiple-organ failure syndrome in acute pancreatitis. Its pathogenesis and treatment. Recenti Prog Med. 1995;86(2):81-5.

8. Death from acute pancreatitis. M.R.C. multicentre trial of glucagon and aprotinin. Lancet. 1977;2 (8039):632-5.

9. Yadav D, Timmons L, Benson JT, Dierkhising RA, Chari ST. Incidence, prevalence, and survival of chronic pancreatitis: a population-based study. Am J Gastroenterol. 2011;106(12):2192-9.

10. Saluja A, Dudeja V, Dawra R, Sah RP. Early Intraacinar events in pathogenesis of pancreatitis. Gastroenterol. 2019;156(7):1979-93.

11. Hietaranta AJ, Saluja AK, Bhagat L, Singh VP, Song AM, Steer ML. Relationship between NF-kappaB and trypsinogen activation in rat pancreas after supramaximal caerulein stimulation. Biochem Biophys Res Commun. 2001;280(1):388-95.

12. Whitcomb DC, Gorry MC, Preston RA. Hereditary pancreatitis is caused by a mutation in the cationic trypsinogen gene. Nat Genet. 1996;14(2):141-5.

13. de Beaux AC, Palmer KR, Carter DC. Factors influencing morbidity and mortality in acute pancreatitis; an analysis of 279 cases. Gut. 1995;37 (1):121-6.

14. Agarwal N, Pitchumoni CS. Assessment of severity in acute pancreatitis. Am J Gastroenterol. 1991;86 (10):1385-91.

15. Gislason H, Horn A, Hoem D. Acute pancreatitis in Bergen, Norway. A study on incidence, etiology and severity. Scand J Surg. 2004;93(1):29-33.

16. Funnell IC, Bornman PC, Weakley SP, Terblanche J, Marks IN. Obesity: an important prognostic factor in acute pancreatitis. Br J Surg. 1993;80(4):484-6.

17. Kelly TR. Gallstone pancreatitis: the timing of surgery. Surg. 1980;88(3):345-50.

18. Acosta MJ, Rossi R, Ledesma CL. The usefulness of stool screening for diagnosing cholelithiasis in acute pancreatitis. A description of the technique. Am $\mathbf{J}$ Dig Dis. 1977;22(2):168-72.

19. Acosta JM, Ledesma CL. Gallstone migration as a cause of acute pancreatitis. N Engl J Med. 1974;290 (9):484-7.

20. Whitcomb DC. Clinical practice. Acute pancreatitis. N Engl J Med. 2006;354(20):2142-50.

21. Acosta JM, Pellegrini CA, Skinner DB. Etiology and pathogenesis of acute biliary pancreatitis. Surg. 1980; 88(1):118-25.

22. Kingsnorth A, O'Reilly D. Acute pancreatitis. Bmj. 2006;332(7549):1072-6. 
23. Smotkin J, Tenner S. Laboratory diagnostic tests in acute pancreatitis. J Clin Gastroenterol. 2002;34(4): 459-62.

24. Clavien PA, Robert J, Meyer P. Acute pancreatitis and normoamylasemia. Not an uncommon combination. Ann Surg. 1989;210(5):614-20.

25. Spechler SJ, Dalton JW, Robbins AH. Prevalence of normal serum amylase levels in patients with acute alcoholic pancreatitis. Dig Dis Sci. 1983;28(10):8659.

26. Yadav D, Agarwal N, Pitchumoni CS. A critical evaluation of laboratory tests in acute pancreatitis. Am J Gastroenterol. 2002;97(6):1309-18.

27. Tietz NW, Shuey DF. Lipase in serum-the elusive enzyme: an overview. Clin Chem. 1993;39(5):74656.

28. Werner J, Feuerbach S, Uhl W, Büchler MW. Management of acute pancreatitis: from surgery to interventional intensive care. Gut. 2005;54(3):42636.

29. Kylänpää-Bäck ML, Kemppainen E, Puolakkainen P. Comparison of urine trypsinogen-2 test strip with serum lipase in the diagnosis of acute pancreatitis. Hepatogastroenterol. 2002;49(46):1130-4.

30. Petersson U, Appelros S, Borgström A. Different patterns in immunoreactive anionic and cationic trypsinogen in urine and serum in human acute pancreatitis. Int J Pancreatol. 1999;25(3):165-70.

31. Whitcomb DC. Value of genetic testing in the management of pancreatitis. Gut. 2004;53(11):17107.

32. Kemppainen E, Hedström J, Puolakkainen P. Increased serum trypsinogen 2 and trypsin 2-alpha 1 antitrypsin complex values identify endoscopic retrograde cholangiopancreatography induced pancreatitis with high accuracy. Gut. 1997;41(5): 690-5.

33. Kemppainen EA, Hedström JI, Puolakkainen PA, et al. Rapid measurement of urinary trypsinogen-2 as a screening test for acute pancreatitis. N Engl J Med. 1997;336(25):1788-93.

34. Neoptolemos JP, Hall AW, Finlay DF, Berry JM, Carr-Locke DL, Fossard DP. The urgent diagnosis of gallstones in acute pancreatitis: a prospective study of three methods. Br J Surg. 1984;71(3):230-3.
35. Tenner S, Dubner H, Steinberg W. Predicting gallstone pancreatitis with laboratory parameters: a meta-analysis. Am J Gastroenterol. 1994;89(10): 1863-6.

36. Ammori BJ, Boreham B, Lewis P, Roberts SA. The biochemical detection of biliary etiology of acute pancreatitis on admission: a revisit in the modern era of biliary imaging. Pancreas. 2003;26(2):e32-5.

37. Dholakia K, Pitchumoni CS, Agarwal N. How often are liver function tests normal in acute biliary pancreatitis?. J Clin Gastroenterol. 2004;38(1):81-3.

38. Griffin N, Wastle ML, Dunn WK, Ryder SD, Beckingham IJ. Magnetic resonance cholangiopancreatography versus endoscopic retrograde cholangiopancreatography in the diagnosis of choledocholithiasis. Eur J Gastroenterol Hepatol. 2003;15(7):809-13.

39. Kejriwal R, Liang J, Anderson G, Hill A. Magnetic resonance imaging of the common bile duct to exclude choledocholithiasis. ANZ J Surg. 2004;74(8):619-21.

40. Kats J, Kraai M, Dijkstra AJ, et al. Magnetic resonance cholangiopancreaticography as a diagnostic tool for common bile duct stones: a comparison with ERCP and clinical follow-up. Dig Surg. 2003;20(1):32-7.

41. Topal B, Van de Moortel M, Fieuws S. The value of magnetic resonance cholangiopancreatography in predicting common bile duct stones in patients with gallstone disease. Br J Surg. 2003;90(1):42-7.

42. Aubé C, Delorme B, Yzet T. MR cholangiopancreatography versus endoscopic sonography in suspected common bile duct lithiasis: a prospective, comparative study. Am J Roentgenol. 2005;184(1):55-62.

Cite this article as: Alghamdi M, Alrashdi AH, Almaghamsi AH, Alamri AE, Abdulrashid BA, Sabban ZM, et al. Biochemical markers in differentiating gallstones pancreatitis from nongallstone pancreatitis. Int J Community Med Public Health 2021;8:890-4. 